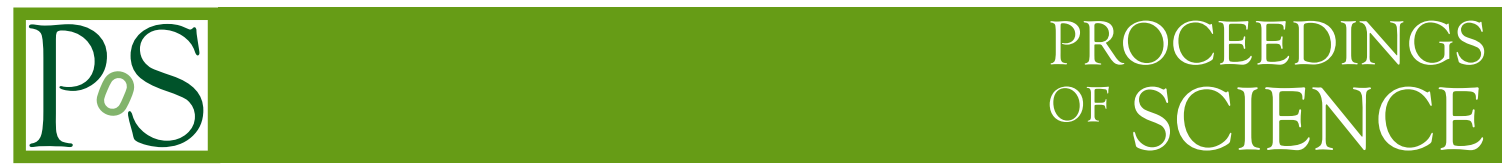

\title{
Status and progress on the CMS Phase-II detector upgrades
}

\section{Nadja Strobbe* on behalf of the CMS Collaboration}

University of Minnesota,

Minneapolis, MN, USA

E-mail: nadja.strobbe@cern.ch

The CMS experiment has an ambitious Phase-II upgrade plan, which includes nearly all parts of the detector. Key aspects include increased channel count and associated bandwidth, improved radiation hardness, and the addition of precision timing. An overview of the upgrade along with a discussion on the current status and recent progress is presented. Overall, excellent progress has been made in the past year, although some delays due to the Covid-19 pandemic were inevitable.

The Ninth Annual Conference on Large Hadron Collider Physics - LHCP2021

7-12 June 2021

Online

${ }^{*}$ Speaker 


\section{Introduction}

The CMS Phase-II detector upgrades [1] ensure that the CMS experiment [2] will maintain excellent object reconstruction efficiency and overall physics performance into the High Luminosity (HL) LHC era. New capabilities will be added to further enhance the reach of the experiment. The CMS Phase-II upgrade has a large scope, essentially building a brand new detector, and pushes detector technologies for high energy physics to a new level. The upgraded detector will provide precise time information using active materials and frontend electronics that can withstand the harsh radiation environment of the HL-LHC. To reduce occupancy in high-pileup conditions, the upgraded detector will feature an increased channel granularity, which is matched by an increase in bandwidth to allow for the readout of this extra data. The trigger latency will also be made longer so that the trigger algorithms can make full use of the extra channel information, which will also include information from the tracking detectors. A short overview and recent progress of the various CMS upgrade projects is provided in the following sections.

\section{Tracker}

Both the Inner and Outer Tracker [3] will be upgraded ahead of HL-LHC to ensure that the tracking performance will be maintained or even improved compared to Run-2 levels. The upgraded tracker features a reduced material budget and an extended acceptance. The Inner Tracker will consist of $2 \times 10^{9}$ silicon pixels arranged in about 4000 modules and several layers, for a total of $4.9 \mathrm{~m}^{2}$ of silicon. The Outer Tracker will be composed of 170 million silicon macro-pixels and 42 million silicon strips in 13,200 modules across several double layers, for a total of $192 \mathrm{~m}^{2}$ of silicon. The silicon sensors will be read out using custom ASICs. Crucially, the Outer Tracker will provide track "stubs" to the track finding system in the Level-1 trigger.

The baseline planar sensors for the Inner Tracker have been radiation tested and are proven to work up to $2 \times 10^{16} \mathrm{~cm}^{-2} 1-\mathrm{MeV}$ neutron-equivalent fluence, a factor of 10 higher than the specifications of the silicon sensors used in the current CMS pixel detector. The inner-most sensor layer is expected to be exposed to even higher radiation levels, and the plan is to replace this layer during Long Shutdown 5. Major progress has also been made on the overall system design, including services. For example, the fiber routing has been redesigned to reduce radiation-induced attenuation. On the Outer Tracker side, the sensor production has started, with the first $5 \%$ batch received. Quality was found to be excellent overall. Final prototypes for the Outer Tracker modules have been tested and the full readout chain was demonstrated.

\section{Endcap Calorimeter}

The existing CMS endcap electromagnetic and hadronic calorimeters will be replaced with an integrated 47-layer sampling calorimeter [4]. The first 26 layers are the electromagnetic section (CE-E) where $\mathrm{Pb}, \mathrm{CuW}$ and $\mathrm{Cu}$ is used as absorber material. The latter 21 layers with steel and $\mathrm{Cu}$ as absorber form the hadronic section $(\mathrm{CE}-\mathrm{H})$. The active material choice depends on the radiation levels: 8-inch hexagonal silicon sensors with 0.5 and $1 \mathrm{~cm}^{2}$ cell sizes are used in the high radiation areas (all of CE-E and part of CE-H), whereas plastic scintillator tiles, ranging in size from 
$23 \times 23 \mathrm{~mm}^{2}$ to $55 \times 55 \mathrm{~mm}^{2}$, with on-tile silicon photomultipliers (SiPM) are used in lower radiation areas at the back of the $\mathrm{CE}-\mathrm{H}$. The upgraded endcap calorimeter will be a 5D imaging calorimeter, providing highly granular spatial information, a large dynamic range for energy measurements, and timing information to tens of picoseconds.

The longitudinal sampling was recently adjusted to account for realistic absorber tolerances, removing 3 layers from the detector, with minimal impact on physics performance. Significant progress was made on the frontend electronics design and system integration. The system will include the Rafael chip for clock distribution, will no longer utilize the bpol2V5 for powering, and will no longer use the GBT-SCA in the silicon region. Extensive tests of the prototype silicon sensors have been completed, including a radiation campaign which showed a much improved oxide quality. The scintillator tileboards were tested in the DESY testbeam, and the HGCROCv3 frontend ASIC was received and is currently under test.

\section{MIP Timing Detector}

A brand new addition to the CMS detector is the MIP timing detector [5] that will timestamp every track with $30 \mathrm{ps}$ resolution at the the start of the HL-LHC. The time resolution is expected to remain better than $50 \mathrm{ps}$ even after $4000 \mathrm{fb}^{-1}$ of integrated luminosity. This precise time information will help maintain detector performance by resolving pileup interactions, resulting in improved efficiencies and resolution. It also opens the door for particle identification for heavy ion and b-quark physics, and can assist in searches for long-lived particles. The MIP timing detector uses two distinct technologies. In the barrel sector, LYSO crystal bars will be used in conjunction with SiPMs on each end of the crystals, for a total of about 332,000 channels between the tracker and the electromagnetic calorimeter. In the endcap sector, two layers of low-gain avalanche detectors, for a total of 8.5 million channels, will be situated in front of the endcap calorimeter. These fast silicon sensors will be bump-bonded to custom ASICs for readout.

The barrel design has recently been updated to include thermoelectric coolers that will provide local cooling and in-situ annealing to alleviate the impact of SiPM radiation damage. Pre-series LYSO and SiPM arrays are now available for final characterization. In addition, the TOFHIR2 ASIC first prototype has been fully characterized in the test bench, with test beam data analysis ongoing. For the endcap design, a beam test at Fermilab was completed with a full chain prototype including the ETROC1 ASIC. Using a telescope with 3 layers, a time resolution of 45 ps per hit was obtained, indicating that the performance from the ETROC1's clock distribution, TDC jitter, and TOT time-walk correction were as expected.

\section{L1 Trigger}

The upgraded CMS L1 Trigger system [6] will perform precise physics selection using a global event reconstruction based on enhanced granularity already at the hardware level. It features an increased latency ( $3.8 \mu \mathrm{s}$ to $12.5 \mu \mathrm{s})$ and an increased trigger rate $(100 \mathrm{kHz}$ to $750 \mathrm{kHz})$, with tracking information used at $40 \mathrm{MHz}$. The trigger system will be implemented using a modular and flexible architecture based on the ATCA platform. Four different flavors of ATCA boards are 
under development. The algorithms use tracking info extensively and exploit the complementarity of different object flavors (e.g. standalone versus track-matched or particle flow).

Recently, a simplified trigger menu based on Run 2 triggers was demonstrated. Algorithms are typically developed in software and then converted into firmware using high-level-synthesis, which provides for a much faster turn-around time and makes it possible to develop novel techniques based on machine learning. Excellent correspondence between the firmware and software emulation has been found. Hardware demonstration is ongoing and will be tested with data during Run 3 running. A low error rate was already achieved using asynchronous link operation at $25.78 \mathrm{~Gb} / \mathrm{s}$ and the recovery mechanism was validated. The demonstration plan includes single-board and multiple board tests in integration centers across the globe with a larger scale integration planned at CERN.

\section{Barrel Calorimeter}

The upgrade of the CMS Barrel Calorimeter [7] includes upgraded frontend electronics for the electromagnetic calorimeter and a new, common set of backend electronics for both electromagnetic and hadronic calorimeters. Progress was made on the frontend amplifier chip (CATIA), which now includes improvements to support ADC calibration. The frontend readout board prototyping is progressing, with a test beam in July 2021, and the backend interfaces have been tested with a demonstrator system.

\section{Muon system}

The Phase-II upgrade of the Muon System [8] includes additional muon layers in the CMS endcaps using GEM technology. The first layer, GE-1/1, was already installed during LS2, with the second layer, GE-2/1, to be installed during the Extended Year-End Technical stop in 2023-2024. One chamber for this second layer has been built and tested using prototype electronics. During LS3, a third layer, ME0, will be installed. Here, the mechanical design was completed, and two prototype modules were produced and tested. This upgrade will extend the muon detector coverage to $|\eta|<3$.

\section{Summary}

The CMS Phase-II upgrades are ambitious, and include state-of-the-art precision timing, highgranularity calorimetry, and the inclusion of tracking information in the hardware trigger. Excellent progress was made in many areas during 2020-2021, despite the challenges brought on by the Covid-19 pandemic. Some inevitable delays were incurred, especially in integration tasks and ASIC production. However, the projects are on track to complete the majority of the prototyping during 2021-2022, after which production will start ramping up. There is still a large amount of work ahead, but the CMS collaboration is up to the task!

\section{References}

[1] CMS collaboration, Technical Proposal for the Phase-II Upgrade of the CMS Detector, Tech. Rep. CERN-LHCC-2015-010, LHCC-P-008, CMS-TDR-15-02, Geneva (Jun, 2015). 
[2] CMS collaboration, The CMS experiment at the CERN LHC, JINST 3 (2008) S08004.

[3] CMS collaboration, The Phase-2 Upgrade of the CMS Tracker, Tech. Rep. CERN-LHCC-2017-009, CMS-TDR-014, CERN, Geneva (Jun, 2017).

[4] CMS collaboration, The Phase-2 Upgrade of the CMS Endcap Calorimeter, Tech. Rep. CERN-LHCC-2017-023, CMS-TDR-019, CERN, Geneva (Nov, 2017).

[5] CMS collaboration, A MIP Timing Detector for the CMS Phase-2 Upgrade, Tech. Rep. CERN-LHCC-2019-003, CMS-TDR-020, CERN, Geneva (Mar, 2019).

[6] CMS collaboration, The Phase-2 Upgrade of the CMS Level-1 Trigger, Tech. Rep. CERN-LHCC-2020-004, CMS-TDR-021, CERN, Geneva (Apr, 2020).

[7] CMS collaboration, The Phase-2 Upgrade of the CMS Barrel Calorimeters, Tech. Rep. CERN-LHCC-2017-011, CMS-TDR-015, CERN, Geneva (Sep, 2017).

[8] CMS collaboration, The Phase-2 Upgrade of the CMS Muon Detectors, Tech. Rep. CERN-LHCC-2017-012, CMS-TDR-016, CERN, Geneva (Sep, 2017). 